Article

\title{
Evaluation of Propagation Characteristics Using the Human Body as an Antenna
}

\author{
Jingzhen $\mathrm{Li}^{1}$, Zedong $\mathrm{Nie}^{1}{ }^{1 *} \mathbb{1}$, Yuhang Liu ${ }^{1}$, Lei Wang ${ }^{1}$ (1) and Yang Hao ${ }^{2}$ \\ 1 Shenzhen Institutes of Advanced Technology, Chinese Academy of Sciences, Shenzhen 518055, China; \\ lijz@siat.ac.cn (J.L.); yh.liu2@siat.ac.cn (Y.L.); wang.lei@siat.ac.cn (L.W.) \\ 2 School of Electronic Engineering and Computer Science, Queen Mary College, University of London, \\ London E1 4NS, UK; y.hao@qmul.ac.uk \\ * Correspondence: zd.nie@siat.ac.cn; Tel.: +86-755-8639-2295; Fax: +86-755-8639-2299
}

Received: 1 November 2017; Accepted: 9 December 2017; Published: 11 December 2017

\begin{abstract}
In this paper, an inhomogeneous human body model was presented to investigate the propagation characteristics when the human body was used as an antenna to achieve signal transmission. Specifically, the channel gain of four scenarios, namely, (1) both TX electrode and RX electrode were placed in the air, (2) TX electrode was attached on the human body, and RX electrode was placed in the air, (3) TX electrode was placed in the air, and RX electrode was attached on the human body, (4) both the TX electrode and RX electrode were attached on the human body, were studied through numerical simulation in the frequency range $1 \mathrm{MHz}$ to $90 \mathrm{MHz}$. Furthermore, the comparisons of input efficiency, accepted efficiency, total efficiency, absorption power of human body, and electric field distribution of different distances of four aforementioned scenarios were explored when the frequency was at $44 \mathrm{MHz}$. In addition, the influences of different human tissues, electrode position, and the distance between electrode and human body on the propagation characteristics were investigated respectively at $44 \mathrm{MHz}$. The results showed that the channel gain of Scenario 4 was the maximum when the frequency was from $1 \mathrm{MHz}$ to $90 \mathrm{MHz}$. The propagation characteristics were almost independent of electrode position when the human body was using as an antenna. However, as the distance between TX electrode and human body increased, the channel gain decreased rapidly. The simulations were verified by experimental measurements. The results showed that the simulations were in agreement with the measurements.
\end{abstract}

Keywords: propagation characteristics; human body; antenna; inhomogeneous model; simulation and measurement

\section{Introduction}

With the development of radio frequency (RF) technology and body sensor network (BSN), the interaction of RF electromagnetic field with the human body has been an active research topic for many years [1,2]. Parts of these studies are focused on the electromagnetic power absorption by the whole body or parts of the body [3-7]. These studies have provided a comprehensive analysis between the power absorption and human body, which are helpful to understand the effects of RF radiation on the human body.

On the other hand, considering the fact that the human tissues are lossy media [8-10], the electric current and electric field are induced in the human body when the human body is exposed to the RF electromagnetic field [11,12]. Therefore, besides the electromagnetic power absorption in human body, the human body may be used itself as an antenna or medium for signal transmission. In recent years, some researchers have demonstrated the propagation characteristics using the human body itself as the transmission medium, which is referred to as human body communication (HBC) or intra-body communication (IBC) [13-17]. However, in the above studies, the transmitter and receiver of HBC are 
acquired to be located on the same human body due to the fact that the electromagnetic field the human body in HBC technology. In other words, it is indicated that the above studies are mainly suitable for the signal transmission in the same individual rather than among different individuals. Thus far, few researchers have tried to characterize the human body as an antenna to achieve the signal transmission among different individuals. In [18], the authors demonstrated the feasibility of energy harvesting from ambient electromagnetic wave using the human body as a receiving antenna. However, only the power spectral density was acquired in the paper, and therefore the propagation characteristics were still not clear when the human body was used as an antenna. In [19], the human-body equivalent monopole antenna, which was made by different combinations of the rectangular blocks, was proposed as a human surrogate. Furthermore, the induced ankle current of model was measured in the frequency range $30 \mathrm{MHz}$ to $100 \mathrm{MHz}$ when the model was exposed to the electromagnetic field. In [20], the human body was demonstrated as an equivalent cylindrical monopole antenna which was grounded on a highly conductive ground plane. The reflection coefficients of antenna were investigated through the finite-difference time-domain (FDTD) algorithm when the frequency was from $10 \mathrm{MHz}$ to $110 \mathrm{MHz}$. In addition, the human body was used as an antenna to achieve the wireless implant communication among different individuals in [21]. Specifically, a toroidal inductor at the cross section of the ankle was used to excite electromotive force inside the human body when the frequency was from $1 \mathrm{MHz}$ to $70 \mathrm{MHz}$. In addition, the receiving toroidal inductor was at the ankle of the other individual. As previously mentioned [19-21], it is indicated that the human body can be represented by a monopole antenna to study the propagation characteristics. However, it requires that the human body stands on a metallic plane or ground plane. Meanwhile, the transmitter and receiver should be fixed on the bottom of the human body (i.e., ankle or sole) which corresponds to the feed point of monopole antenna when the human body is modeled as a monopole antenna. Once the position of transmitter or receiver is changed, the human-body equivalent model of monopole antenna may be unavailable. Furthermore, thus far, the factors affecting propagation characteristics are still ambiguous when the human body is used as an antenna. Therefore, in order to better understand the characteristics of human body when it is utilized as an antenna and provides a prospective communication solution for BSN, a comprehensive investigation is necessary and important.

In this paper, we mainly focused on the propagation characteristics when the human body was employed as an antenna to achieve the signal transmission among different individuals. Specifically, different from the previous investigations which the human body is regarded as a monopole antenna and the power is injected into the human ankle or sole by using current directly, the power is coupled into the human body through electrode in this paper, and then the power is radiated out of the body when the human body is utilized as a transmitting antenna. Similarly, the power is coupled into the receiver when the human body is used as a receiving antenna. The advantage of this method is that the positions of the transmitter and the receiver are alterable, so they are no longer limited to the human ankle or sole. The contribution and originality of this paper was summarized as follows. Firstly, the propagation characteristics were investigated when the human body was utilized as an antenna in the frequency range from $1 \mathrm{MHz}$ to $90 \mathrm{MHz}$. Secondly, the factors affecting propagation characteristics, including the different human tissues, the electrode position on the human body, and the distance between electrode and human body were studied in this paper.

The remainder of this paper is organized as follows. In Section 2, we will present the inhomogeneous human body model and the simulation setup under different conditions. In Section 3 , the experimental measurement will be reported. Section 4 discusses and gives a detailed analysis of both simulation and measurement results. Finally, the conclusions are drawn in Section 5. 


\section{Simulation Setup}

\subsection{Human Body Model Proposed}

In this work, an inhomogeneous human body model, which consists of head, chest, abdomen, upper arm, forearm, thigh, and lower leg, was proposed to explore the propagation characteristics when the human body was used as an antenna. As shown in Figure 1, the height of the human body model was $1698 \mathrm{~mm}$, which corresponded with the average height of an adult [22].The tissue layers and thicknesses of different body parts in this model are listed in Table 1 [23].
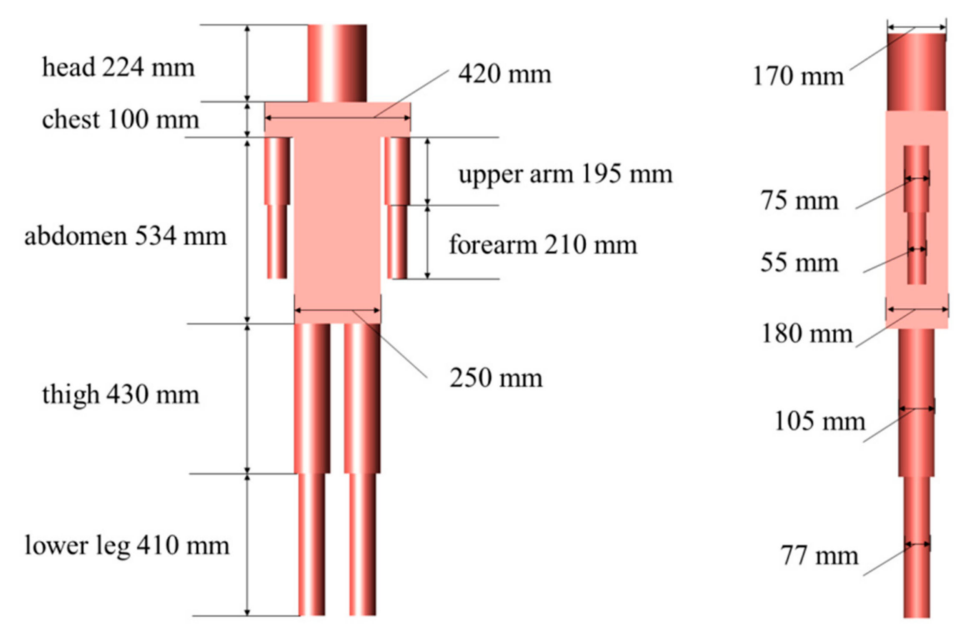

Figure 1. The dimension and body parts of human body model.

Table 1. The tissue layers and thicknesses of different body parts in human body model (Unit: $\mathrm{mm}$ ).

\begin{tabular}{ccccccc}
\hline Body Parts & Skin & Fat & Muscle & Bone & Grey Matter & Heart \\
\hline head & 4 & - & 9.5 & 20.5 & 51 & - \\
chest & 2 & 4 & 38 & 46 & - & - \\
abdomen & 2 & 7 & 25 & - & - & 56 \\
upper arm & 2 & 6.1 & 20.3 & 9.1 & - & - \\
forearm & 2 & 4.3 & 14.9 & 6.3 & - & - \\
thigh & 2 & 8.8 & 28.7 & 13 & - & - \\
lower leg & 2 & 6.3 & 20.8 & 9.4 & - & - \\
\hline
\end{tabular}

\subsection{Simulation Setup for Four Scenarios}

In order to better evaluate the propagation characteristics when the human body was utilized as an antenna, as demonstrated in Figure 2, four scenarios were considered in this section. Namely, in Scenario 1, both the transmitter (TX electrode and GND electrode) and receiver (RX electrode and GND electrode) were exposed to the air. In Scenario 2, the TX electrode of the transmitter was attached to the human body, and GND electrode was floated, and then the human body was regarded as a transmitting antenna to transmit signal. Additionally, the signal was received by the RX electrode directly which was exposed to the air. The setup of Scenario 3 was in opposition to that of Scenario 2 . In Scenario 4, the TX electrode and RX electrode were attached on a different human body, and the GND electrodes were suspended in the air. The numerical simulations of four aforementioned scenarios were performed by using electromagnetic software XFDTD which is based on FDTD method. The size of TX electrode, RX electrode, and GND electrode in the simulation was $34 \mathrm{~mm} \times 34 \mathrm{~mm} \times 0.2 \mathrm{~mm}$. The distance between TX electrode and GND electrode was $26.2 \mathrm{~mm}$. Similarly, the distance between RX electrode and GND electrode was also $26.2 \mathrm{~mm}$. The TX electrode was excited by a voltage source, and the waveform of voltage source was a broadband signal. Meanwhile, there was a resistance load 
between the RX electrode and GND electrode. The distance between the transmitter and receiver in all four scenarios was $800 \mathrm{~mm}$. The channel gain between the transmitter and receiver was investigated when the frequency was from $1 \mathrm{MHz}$ to $90 \mathrm{MHz}$. Considering that the resonant frequency of the human body was mainly between $40 \mathrm{MHz}$ and $60 \mathrm{MHz}$ [12,20], the frequency which was from $1 \mathrm{MHz}$ to $90 \mathrm{MHz}$ was selected as the research object in this paper because it could include the resonant frequency of the human body.

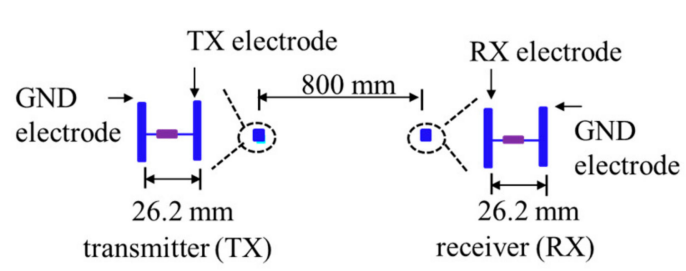

(a)

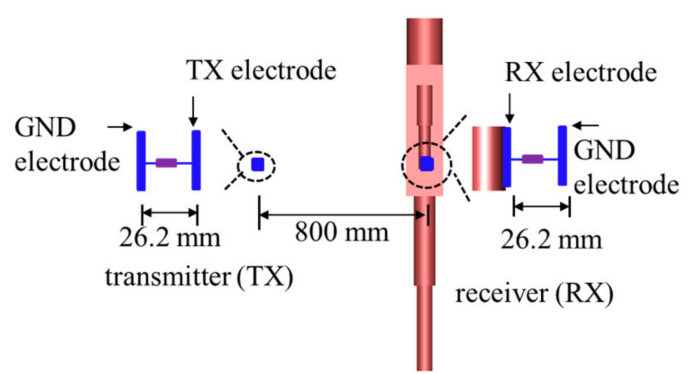

(c)
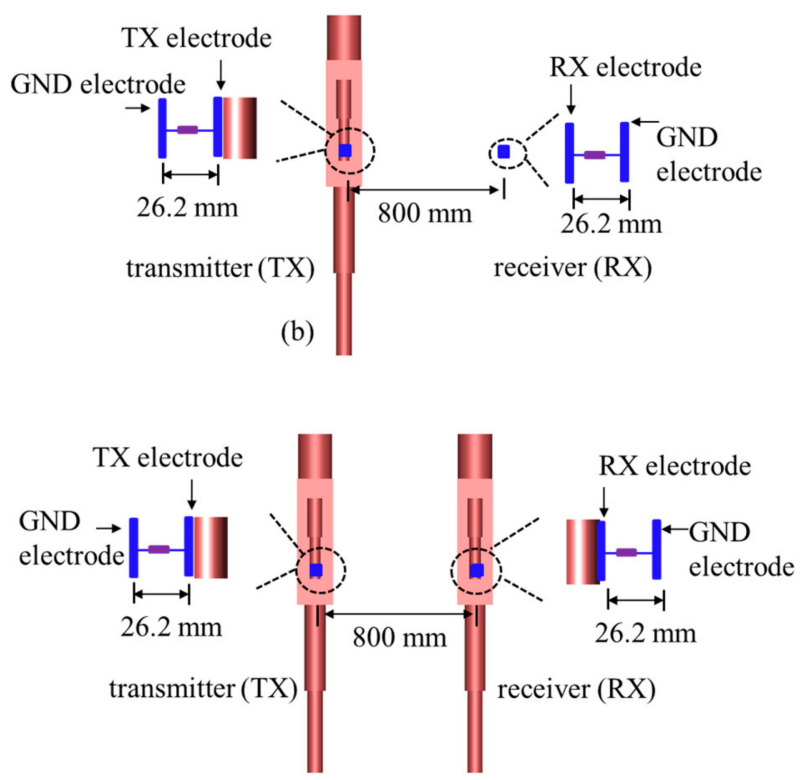

(d)

Figure 2. The simulation setup of four scenarios: (a) Scenario 1: TX electrode without human body, RX electrode without human body; (b) Scenario 2: TX electrode with human body, RX electrode without human body; (c) Scenario 3: TX electrode without human body, RX electrode with human body; (d) Scenario 4: TX electrode with human body, RX electrode with human body.

\subsection{Simulation Setup for the Influence of Electrode Position}

In this section, we mainly concentrated on the influence of electrode position when the human body was employed as an antenna. As will be shown later, the propagation characteristics of Scenario 2 and Scenario 3 are quite similar. Therefore, to save the computation, the influence of electrode position under Scenario 3 and Scenario 4 were studied respectively in this section. Figure 3a shows the positions of electrode. Specifically, For Scenario 3, the TX electrode and GND electrode were placed in the air (F), and the RX electrode was attached on the different parts of the human body, which were forearm (A), abdomen (B), upper arm (C), thigh (D), and back (E). Similarly, for Scenario 4, the positions of TX electrode and RX electrode were located on the different parts of the human body. The TX electrode was excited by a voltage source. The waveform of voltage source was a sinusoid, of which the frequency was $44 \mathrm{MHz}$. There was a resistance load between the RX electrode and the GND electrode. The convergence of calculation was defined as $-30 \mathrm{~dB}$ in FDTD simulation. 


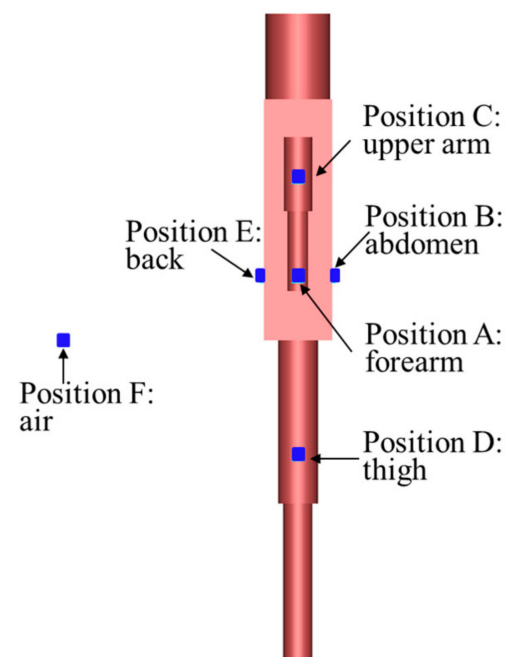

(a)

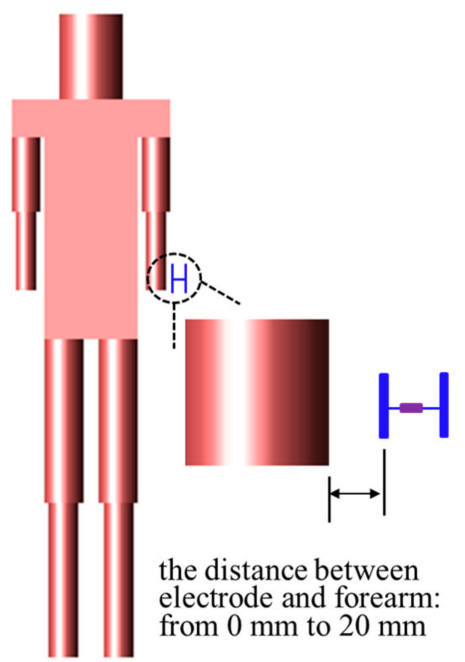

(b)

Figure 3. (a) Simulation setup about the positions of electrode; (b) simulation setup about the distance between electrode and human body.

\subsection{Simulation Setup for the Influence of Distance between Electrode and Human Body}

As illustrated in Figure 3b, the influence of the distance between the electrode and the human body was studied in this section. Details of simulation setup are as follows. For Scenario 3, the TX electrode were placed in the air and fixed on the specified position, and the RX electrode was placed on the surface of the human forearm. Furthermore, the distance between the RX electrode and the forearm was variable, which was from $0 \mathrm{~mm}$ to $20 \mathrm{~mm}$. The TX electrode was fed by a voltage source of which the waveform is a $44 \mathrm{MHz}$ sinusoid. In addition, the simulation setup of Scenario 4 was similar to Scenario 3.

\section{Experimental Setup}

\section{Experimental Scenario}

To verify the validity of numerical simulations with an inhomogeneous human body model, experiment measurements were executed in this paper. Written informed consent was obtained from the volunteer before the measurement. As shown in Figure 4, two volunteers stood on the floor, and the distance between the volunteers was consistent with the simulation setup. The TX electrode, RX electrode, GND electrodes were fabricated by the copper. The distance between TX electrode/RX electrode and GND electrode was about $26.2 \mathrm{~mm}$. The TX electrode of transmitter was attached on the forearm of volunteer 1 by the wristband, and the GND electrode of transmitter was exposed to the air. Furthermore, the transmitter was connected to the Port 1 of vector network analyzer (VNA, model number: E5061A, Agilent, CA, USA) by the cable and balun transformer with turn ratio of 1:1 (model number: FTB-1-1, Mini-Circuits, NY, USA). Similarly, the receiver was attached to volunteer 2 and connected to the Port 2 of VNA. The balun transformers were adopted in the experimental measurement for the reason that it could break the connection between the GND electrodes of transmitter and receiver [24,25], which corresponded to the simulation setup. The channel gain between transmitter and receiver was acquired by the VNA at four aforementioned scenarios in the frequency range $1 \mathrm{MHz}$ to $90 \mathrm{MHz}$. Subsequently, the position of the transmitter or receiver was changed, and the channel gain was obtained at $44 \mathrm{MHz}$. Finally, the influence of the distance between electrode and human body was measured by the VNA at $44 \mathrm{MHz}$. 


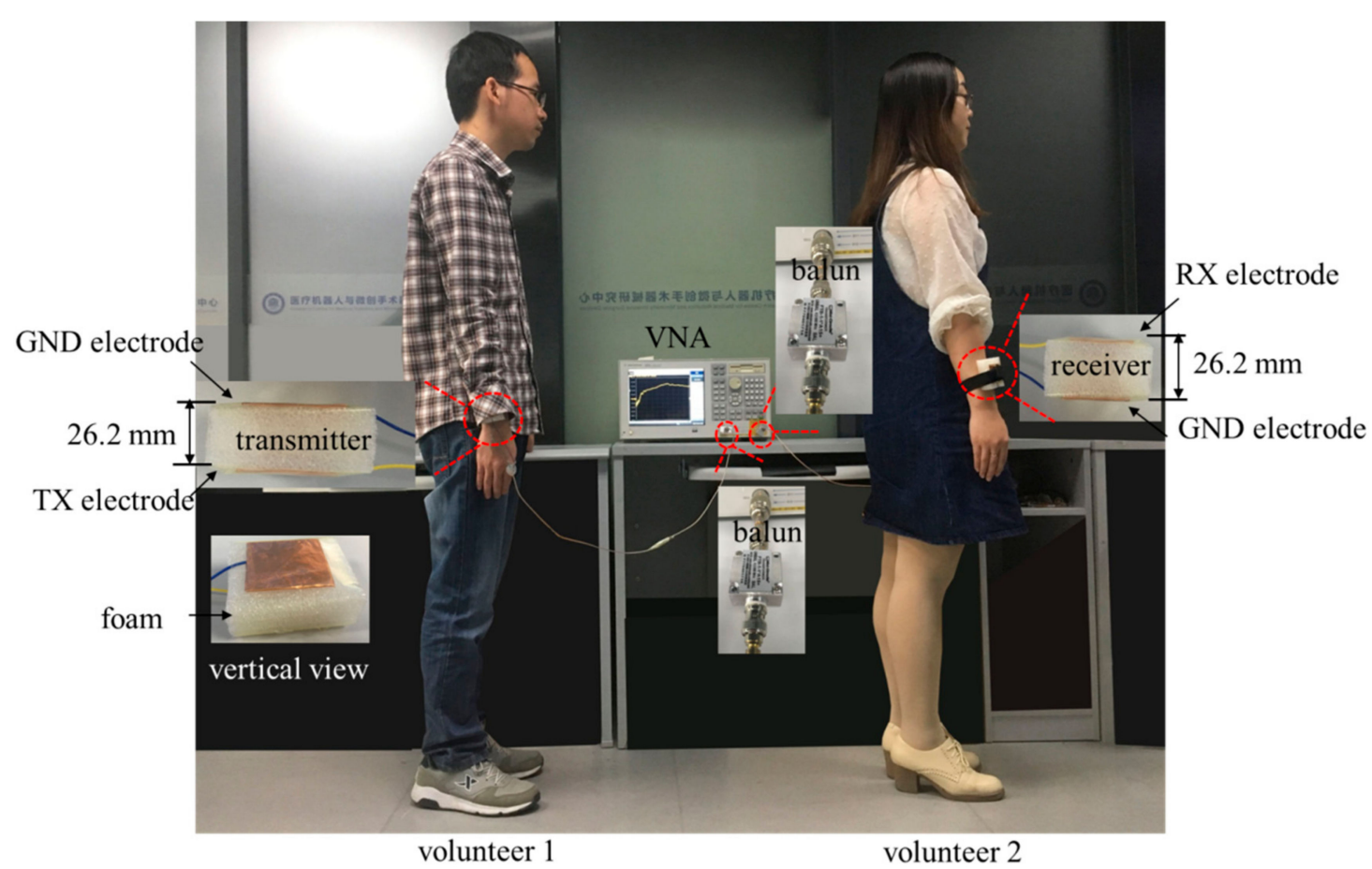

Figure 4. Experimental scenario.

\section{Results and Analysis}

\subsection{Propagation Characteristics of Different Scenarios}

\subsubsection{The Channel Gain of Four Scenarios in the Frequency Range from $1 \mathrm{MHz}$ to $90 \mathrm{MHz}$}

Figure 5 illustrates the channel gain (in $\mathrm{dB}$ ) of four scenarios through simulations and measurements when the frequency was from $1 \mathrm{MHz}$ to $90 \mathrm{MHz}$. It is noteworthy that in order to better understand the propagation characteristics among four scenarios, the simulation results were pre-processed. Specifically, the maximum value of channel gain was set as a reference value, and then the difference between channel gain and reference value were obtained. Similarly, the measurement results were also pre-processed by the above method. According to the simulation results, the gain of all four scenarios had the smallest value at $1 \mathrm{MHz}$. When the frequency was below $65 \mathrm{MHz}$, as the frequency increased, the gain of all four scenarios increased. Additionally, it was interesting to observe that the gain of Scenario 2 and Scenario 3 was almost the same. On the other hand, as shown in Figure 5, the difference among the four scenarios was quite obvious when the frequency was below $65 \mathrm{MHz}$. The gain of Scenario 1 was the minimum, whereas the gain of Scenario 4 was the maximum. For instance, the gain of four scenarios was approximately $-39.81 \mathrm{~dB},-25.48 \mathrm{~dB},-27.77 \mathrm{~dB},-9.56 \mathrm{~dB}$ at $15 \mathrm{MHz}$, respectively. Furthermore, the gain was $-30.85 \mathrm{~dB},-19.60 \mathrm{~dB},-18.51 \mathrm{~dB},-2.99 \mathrm{~dB}$ when the frequency was $50 \mathrm{MHz}$. However, the channel gain of four scenarios was $-24.53 \mathrm{~dB},-13.41 \mathrm{~dB}$, $-12.95 \mathrm{~dB},-4.21 \mathrm{~dB}$ individually at the frequency of $80 \mathrm{MHz}$. In addition, the results showed an agreement between the numerical simulations and experimental measurements for Scenario 2, Scenario 3 and Scenario 4 . However, there was a little deviation for Scenario 1. 


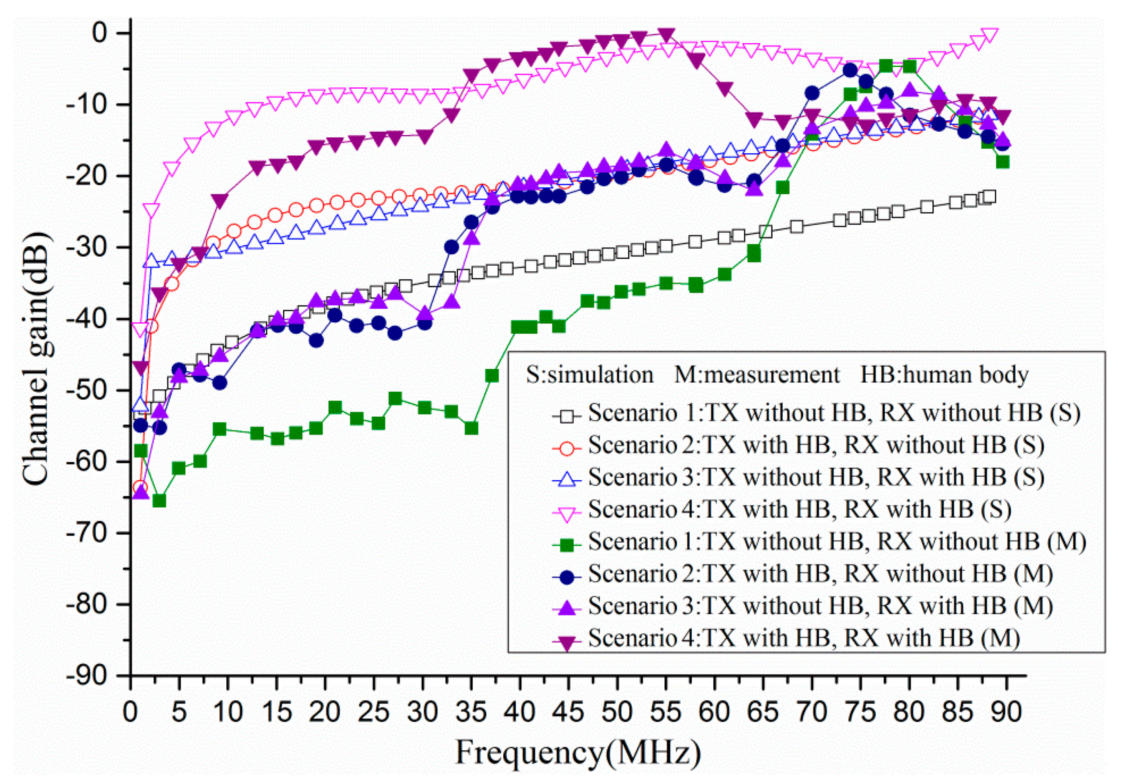

Figure 5. The propagation characteristics of four scenarios in the frequency range $1 \mathrm{MHz}$ to $90 \mathrm{MHz}$.

Figure 6 shows the channel gain (in dB) of different distances (i.e., $400 \mathrm{~mm}, 800 \mathrm{~mm}, 1200 \mathrm{~mm}$, $1600 \mathrm{~mm}$ ) between transmitter and receiver at Scenario 4 when the frequency was from $1 \mathrm{MHz}$ to $90 \mathrm{MHz}$. The simulation results and measurement results were also pre-processed by the aforementioned method. In terms of simulation results, it could be observed that as the distance between transmitter and receiver increased, the channel gain was decreased when the frequency was below $80 \mathrm{MHz}$. On the other hand, it was found that when the distance was beyond $800 \mathrm{~mm}$, the difference of channel gain among different distances became small. For instance, the channel gain was more than $-1.58 \mathrm{~dB}$ at $44 \mathrm{MHz}$ when the distance was $400 \mathrm{~mm}$. However, the channel gain was $-9.77 \mathrm{~dB},-13.39 \mathrm{~dB},-16.01 \mathrm{~dB}$ respectively at $44 \mathrm{MHz}$ when the distance was $800 \mathrm{~mm}, 1200 \mathrm{~mm}$, $1600 \mathrm{~mm}$. Thus, the distance between transmitter and receiver was taken as $800 \mathrm{~mm}$ in this paper.

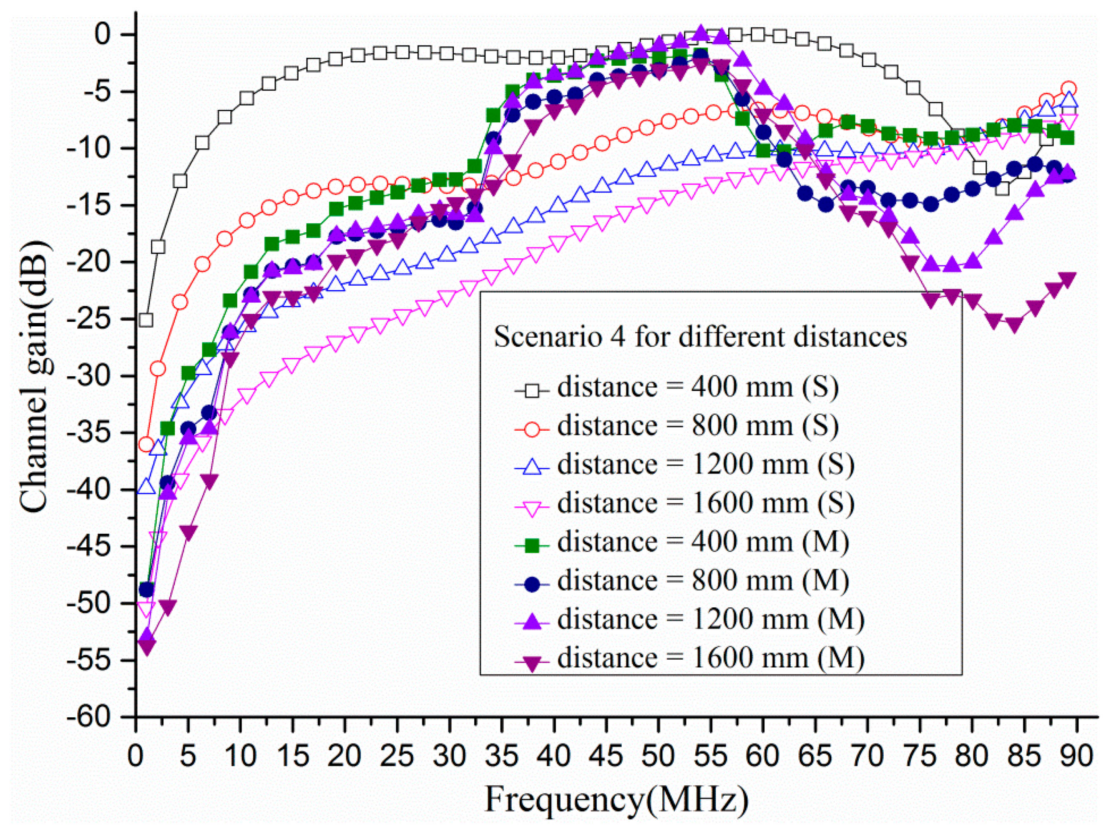

Figure 6. The propagation characteristics of different distance at Scenario 4. 


\subsubsection{The Comparison of Four Scenarios at $44 \mathrm{MHz}$}

To better understand the propagation characteristics when the human body was employed as an antenna, the input power, absorption power in human body, accepted power, input efficiency, accepted efficiency, and total efficiency of four scenarios were investigated when the frequency was at $44 \mathrm{MHz}$. The frequency of $44 \mathrm{MHz}$ was selected as the research object for the following reasons. Firstly, according to $[12,19,20]$, it was found that the human body resonated between $40 \mathrm{MHz}$ and $60 \mathrm{MHz}$ when it was exposed to the electromagnetic wave. Secondly, the height of the human body model in this paper was $1698 \mathrm{~mm}$, which corresponded to the one-quarter wavelength when the frequency was $44 \mathrm{MHz}$. In this paper, the available power is the power which can be delivered by the voltage source given a conjugate impedance match between the simulation spaces. The input power is defined as the power delivered by a voltage source into the simulation space practically. It is worth noting that the available power was set as $1 \mathrm{~W}$ in the simulations. The input efficiency, accepted efficiency, and total efficiency are calculated as Equations (1)-(3), respectively. Table 2 lists the comparison of four scenarios.

$$
\begin{gathered}
\text { input efficiency }=\frac{\text { input power }}{\text { available power }} \times 100 \% \\
\text { accepted efficiency }=\frac{\text { accepted power }}{\text { input power }} \times 100 \% \\
\text { total efficiency }=\text { input efficiency } \times \text { accepted efficiency }
\end{gathered}
$$

Table 2. Comparison of four scenarios at $44 \mathrm{MHz}$.

\begin{tabular}{ccccc}
\hline & Scenario 1 & Scenario 2 & Scenario 3 & Scenario 4 \\
\hline input power(W) & $9.22 \times 10^{-6}$ & 0.0753 & $4.90 \times 10^{-7}$ & 0.07529 \\
total absorption power (W) & - & 0.05989 & $3.312 \times 10^{-7}$ & 0.05991 \\
TX absorption power (W) & - & 0.05989 & - & 0.05989 \\
RX absorption power (W) & - & - & $3.312 \times 10^{-7}$ & 0.00002 \\
accepted power (W) & $4.55 \times 10^{-10}$ & $6.13 \times 10^{-9}$ & $6.06 \times 10^{-9}$ & $2.378 \times 10^{-7}$ \\
input efficiency & $9.22 \times 10^{-4} \%$ & $7.53 \%$ & $4.90 \times 10^{-5} \%$ & $7.53 \%$ \\
accepted efficiency & $4.90 \times 10^{-3} \%$ & $8.14 \times 10^{-6} \%$ & $1.24 \%$ & $3.16 \times 10^{-4} \%$ \\
total efficiency & $4.55 \times 10^{-8} \%$ & $6.13 \times 10^{-7} \%$ & $6.05 \times 10^{-7 \%}$ & $2.38 \times 10^{-5} \%$ \\
\hline
\end{tabular}

As listed in Table 2, it could be observed that the input efficiency of Scenario 1 was just $9.22 \times 10^{-4 \%}$, which was $9.22 \times 10^{-6} \mathrm{~W}$ when the TX electrode and RX electrode were placed in the air. The total efficiency of Scenario 1 was just $4.55 \times 10^{-8 \%}$. Compared with Scenario 1 , the input efficiency of Scenario 2 was increased to $7.53 \%$ when the TX electrode was attached to the human body. Therefore, it seems to indicate that more power could be injected into the simulation space when the human body was used as a transmitting antenna. Part of the power was absorbed by the human, and the other power was radiated out of the human body. The power was accepted by the RX electrode directly in Scenario 2, and the accepted efficiency was just $8.14 \times 10^{-6 \%}$. Thus, the total efficiency of the Scenario was $6.13 \times 10^{-7} \%$. The input efficiency of Scenario 3 was $4.90 \times 10^{-5} \%$, which was similar to Scenario 1 . However, the accepted efficiency was up to $1.24 \%$ when the RX electrode was attached to the human body. Thus, it was evident that the human body could harvest the power when it was used as a receiving antenna. The total efficiency of Scenario 3 was about $6.05 \times 10^{-7} \%$. For Scenario 4 , the input efficiency was up to $7.53 \%$. In addition, the accepted efficiency and total efficiency was $3.16 \times 10^{-4} \%$ and $2.38 \times 10^{-5} \%$, respectively. Therefore, compared with other scenarios, the total efficiency of Scenario 4 was the greatest. To sum up, it is helpful to improve the input efficiency and accepted efficiency when the human body is used as an antenna. 


\subsubsection{Electric Field Distribution of Different Distances at $44 \mathrm{MHz}$}

To intuitively analyze and compare the performances of four scenarios, the electric field distribution was studied in this section. Figure 7 demonstrates the electric field distribution of different distances for four scenarios when the frequency was $44 \mathrm{MHz}$. The original point $(0 \mathrm{~mm})$ was set as the position of transmitter. The distance from transmitter to receiver was $800 \mathrm{~mm}$. The reference value of electric field intensity (EFI) was $500 \mathrm{~V} / \mathrm{m}$ (i.e., $0 \mathrm{~dB}$ ). As shown in the 3rd frame of Scenario 1 , the EFI of TX electrode was approximately $-20 \mathrm{~dB}$, and the EFI near the TX electrode was from $-32 \mathrm{~dB}$ to $-64 \mathrm{~dB}$. As the distance increased, the EFI decreased gradually. The EFI was from $-72 \mathrm{~dB}$ to $-76 \mathrm{~dB}$ when the distance was $800 \mathrm{~mm}$. Moreover, the EFI declined to $-80 \mathrm{~dB}$ at the distance of $1075 \mathrm{~mm}$. Different from the electric field distribution in Scenario 1, the EFI of Scenario 2 in the 3rd frame was from $-8 \mathrm{~dB}$ to $-56 \mathrm{~dB}$. Meanwhile, it could be observed that the EFI was almost the same when the distance was $-90 \mathrm{~mm}$ and $105 \mathrm{~mm}$. In addition, the EFI was quite similar when the distance was $-290 \mathrm{~mm}$ and $25 \mathrm{~mm}$. Thus, it was inferred that the power which is radiated out of the human body was omnidirectional. Compared with Scenario 1, the EFI was more than $-56 \mathrm{~dB}$ when the distance was $800 \mathrm{~mm}$, which indicated that the human body could be considered an effective transmitting antenna. On the other hand, it was interesting to observe that the electric field distribution of Scenario 3 was quite similar to Scenario 1 when the distance was below $415 \mathrm{~mm}$, which was because both the TX electrodes of Scenario 1 and Scenario 3 were not attached to the human body. However, the EFI of Scenario 3 was greater than Scenario 1 when the distance was over $710 \mathrm{~mm}$, which might be caused by the effect of the human body. As shown in the 8th, 9th and 10th frame of Scenario 3, the EFI around the human body was mainly from $-56 \mathrm{~dB}$ to $-80 \mathrm{~dB}$. The electric field distribution of Scenario 4 was almost the same as Scenario 2 when the distance was below $415 \mathrm{~mm}$. Because of the influence of the human body on the RX electrode, the EFI of Scenario 4 was greater than Scenario 2 when the distance was over $575 \mathrm{~mm}$. The EFI of Scenario 4 was from $-40 \mathrm{~dB}$ to $-64 \mathrm{~dB}$ when the distance was $800 \mathrm{~mm}$. Therefore, to sum up, the EFI is prominently enhanced when the TX electrode and RX electrode are attached to the human body.

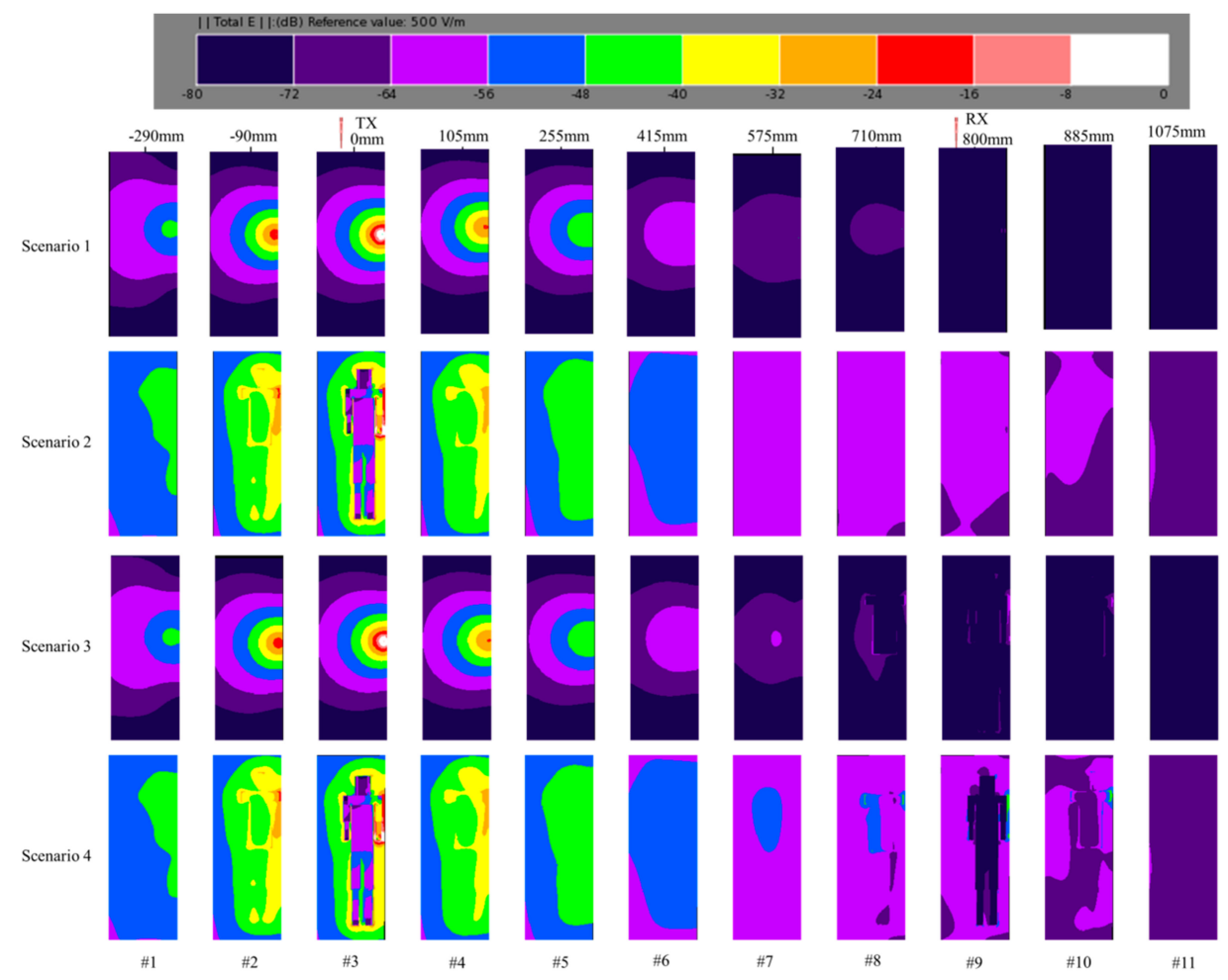

Figure 7. Electric field distribution of different distances at $44 \mathrm{MHz}$. 


\subsection{Investigation on the Influence of Human Tissues}

It is known that the human body consists of different tissues, and the dielectric properties (i.e., permittivity and conductivity) of different tissues are different. Thus, the influence of human tissue on propagation characteristics was studied in the paper. Considering that the principal tissues of the human body are skin, fat, muscle, bone, and organ [26,27], the aforementioned tissues were studied at $44 \mathrm{MHz}$ in this paper. Specifically, the human body was modeled as a homogeneous model of skin, fat, muscle, bone, and organ, respectively. Furthermore, in terms of dielectric properties, there is no significant difference between the heart and other organs, such as liver, kidney, and so on [10]. Therefore, the dielectric property of the heart was used to represent other organs in this paper. The dielectric properties of different tissues at $44 \mathrm{MHz}$ are listed in Table 3, and the total efficiency of different models are listed in Table 4.

Table 3. The dielectric properties of different tissues at $44 \mathrm{MHz}$.

\begin{tabular}{cccccc}
\hline Biological Tissue & Skin & Fat & Muscle & Bone & Heart \\
\hline relative permittivity & 116.54 & 7.1176 & 80.069 & 18.4 & 124.85 \\
conductivity $(\mathrm{S} / \mathrm{m})$ & 0.38954 & 0.034347 & 0.67297 & 0.055928 & 0.63689 \\
\hline
\end{tabular}

Table 4. Total efficiency of different human body models using as an antenna at $44 \mathrm{MHz}$.

\begin{tabular}{ccccccc}
\hline Total Efficiency & Skin Model & Fat Model & Muscle Model & Bone Model & Heart Model & Inhomogeneous Model \\
\hline Scenario 3 & $6.21 \times 10^{-7} \%$ & $3.96 \times 10^{-7} \%$ & $7.53 \times 10^{-7} \%$ & $3.79 \times 10^{-7} \%$ & $7.29 \times 10^{-7 \%}$ & $6.05 \times 10^{-7} \%$ \\
Scenario 4 & $2.27 \times 10^{-5} \%$ & $3.80 \times 10^{-6} \%$ & $4.09 \times 10^{-5} \%$ & $3.85 \times 10^{-6} \%$ & $3.65 \times 10^{-5} \%$ & $2.38 \times 10^{-5} \%$ \\
\hline
\end{tabular}

As listed in Table 4 of Scenario 3, the total efficiency was associated with the conductivity and relative permittivity of model. For example, the total efficiency was $6.21 \times 10^{-7 \%}, 7.53 \times 10^{-7 \%}$ and $7.29 \times 10^{-7 \%}$ for skin model, muscle model and heart model. However, the total efficiency of fat model and bone model was relatively small, which was $3.96 \times 10^{-7} \%$ and $3.79 \times 10^{-7} \%$. Furthermore, according to the dielectric properties of human tissues, the skin, muscle and heart are high-water-content tissues, whereas the fat and bone are low-water-content tissues [28,29]. Therefore, it was indicated that the high-water-content tissues played an important role in signal propagation when the human body was utilized as an antenna. In addition, as listed in in Table 4 of Scenario 4 ,

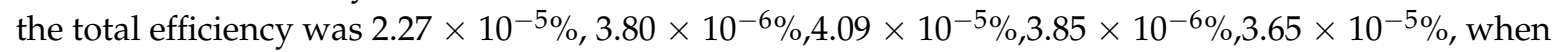
the human body was modeled as skin model, fat model, muscle model, bone model, and heart model, respectively. Thus, it also revealed the same conclusion that the high-water-content tissues, rather than the low-water-content tissues, played an important role in signal propagation.

\subsection{Investigation on the Influence of Electrode Position}

Table 5 lists the channel gain of Scenario 3 at $44 \mathrm{MHz}$ when the TX electrode was placed in the air, and the RX electrode was attached on the different position of human body surface. The channel gain was pre-processed in this section so that we could study the influence of electrode position intuitively. Specifically, the value of channel gain was regarded as a reference value when the RX electrode was attached on the forearm. Subsequently, the differences between channel gain of different positions and reference value were obtained. As listed in Table 5, the channel gain was $0 \mathrm{~dB}, 1.285 \mathrm{~dB}, 2.521 \mathrm{~dB}$, $-0.871 \mathrm{~dB},-0.871 \mathrm{~dB}$, respectively in our simulations. Moreover, the difference between the minimum and maximum values was no more than $3.4 \mathrm{~dB}$. Therefore, it could be inferred that the electrode position had little impact on channel gain when the human body was used as receiving antenna. In addition, the channel gain was $0 \mathrm{~dB},-0.110 \mathrm{~dB},-0.308 \mathrm{~dB},-1.295 \mathrm{~dB},-1.035 \mathrm{~dB}$, respectively in the experimental measurements. The measurements also showed that the channel gain was almost independent of the electrode position. 
Table 5. The influence of electrode position at $44 \mathrm{MHz}$ for Scenario 3.

\begin{tabular}{lccccc}
\hline TX Electrode & Air & Air & Air & Air & Air \\
\hline RX electrode & forearm & upper arm & thigh & abdomen & back \\
simulation $(\mathrm{dB})$ & 0 & 1.285 & 2.521 & -0.871 & -0.871 \\
measurement $(\mathrm{dB})$ & 0 & -0.110 & -0.308 & -1.295 & -1.035 \\
\hline
\end{tabular}

Table 6 demonstrates the channel gain of Scenario 4 at $44 \mathrm{MHz}$ when the TX electrode and RX electrode were placed on the different positions of human body surface. The channel gain was also pre-processed, and the value of channel gain was regarded as a reference value when the TX electrode and RX electrode were attached on the forearms of different humans. From the simulation results, it could be observed that the channel gain was $0 \mathrm{~dB},-0.492 \mathrm{~dB},-1.283 \mathrm{~dB},-1.816 \mathrm{~dB},-1.815 \mathrm{~dB}$, $-1.921 \mathrm{~dB}$, and $-1.921 \mathrm{~dB}$ at different positions. Therefore, it could be concluded that channel gain was also independent of electrode position when the human bodies were using as transmitting antenna and receiving antenna. Additionally, the channel gain was $0 \mathrm{~dB},-0.510 \mathrm{~dB},-0.999 \mathrm{~dB},-1.700 \mathrm{~dB}$, $-2.296 \mathrm{~dB},-3.412 \mathrm{~dB},-3.390 \mathrm{~dB}$ in our measurements.

Table 6. The influence of electrode position at $44 \mathrm{MHz}$ for Scenario 4 .

\begin{tabular}{cccccccc}
\hline TX Electrode & Forearm & Forearm & Forearm & Forearm & Forearm & Abdomen & Back \\
\hline RX electrode & forearm & upper arm & thigh & abdomen & back & abdomen & back \\
simulation $(\mathrm{dB})$ & 0 & -0.492 & -1.283 & -1.816 & -1.815 & -1.921 & -1.921 \\
measurement $(\mathrm{dB})$ & 0 & -0.510 & -0.999 & -1.700 & -2.296 & -3.412 & -3.390 \\
\hline
\end{tabular}

\subsection{Investigation on the Influence of Distance between Electrode and Human Body}

Figure 8 shows the results of channel gain as a function of the distance between RX electrode and human body for Scenario 3. In this section, the value of channel gain was regarded as a reference value when the distance was $0 \mathrm{~mm}$. Subsequently, the differences between channel gain of different distances and reference value were obtained. In terms of the simulation results, the channel gain was $0 \mathrm{~dB}$ when the RX electrode was closely attached on the human body. As the distance increased, the channel gain decreased. The channel gain was declined from $0 \mathrm{~dB}$ to $-7.77 \mathrm{~dB}$ at the distance of $10 \mathrm{~mm}$. Subsequently, the decrease of channel gain became slow while the distance was over $10 \mathrm{~mm}$. The channel gain was $-8.22 \mathrm{~dB},-8.89 \mathrm{~dB},-9.52 \mathrm{~dB},-9.81 \mathrm{~dB}$, and $-10.43 \mathrm{~dB}$ respectively at the distance of $12 \mathrm{~mm}, 14 \mathrm{~mm}, 16 \mathrm{~mm}, 18 \mathrm{~mm}$, and $20 \mathrm{~mm}$. According to the correlation between channel gain and distance, the correlation could be fitted by the quartic polynomial, as shown in Equation (4). In addition, the measurement result also showed the same decreasing trend.

$$
y=-0.478-2.039 \cdot x+0.252 \cdot x^{2}-0.015 \cdot x^{3}+3.169 \times 10^{-4} \cdot x^{4}
$$

where $y$ is the channel gain, and $x$ is the distance between RX electrode and human body.

The channel gain of different distances between electrode and human body at Scenario 4 is plotted in Figure 9. As shown in Figure 9, the changing trend of channel gain was almost the same between simulation and measurement. According to the simulation, the channel gain was $0 \mathrm{~dB}$ when both the TX electrode and RX electrode were closely attached on the human bodies. However, the channel gain decreased sharply as the distance increased. For instance, the channel gain declined from $0 \mathrm{~dB}$ to $-8.78 \mathrm{~dB}$ when the distance was $4 \mathrm{~mm}$. Furthermore, the channel gain was $-13.52 \mathrm{~dB}$ and $-18.49 \mathrm{~dB}$ when the distance was $10 \mathrm{~mm}$ and $20 \mathrm{~mm}$ individually. Thus, it was revealed that the distance had a great impact on the channel gain when the human bodies were used as transmitting antenna and receiving antenna. To better evaluate the influence of different distances on channel gain, 
the relationship between channel gain and the distance was fitted by the polynomial, as shown in Equation (5).

$$
y=-0.605-3.355 \cdot x+0.390 \cdot x^{2}-0.023 \cdot x^{3}+4.833 \times 10^{-4} \cdot x^{4}
$$

where $y$ is the channel gain, and $x$ is the distance between electrode and human body.

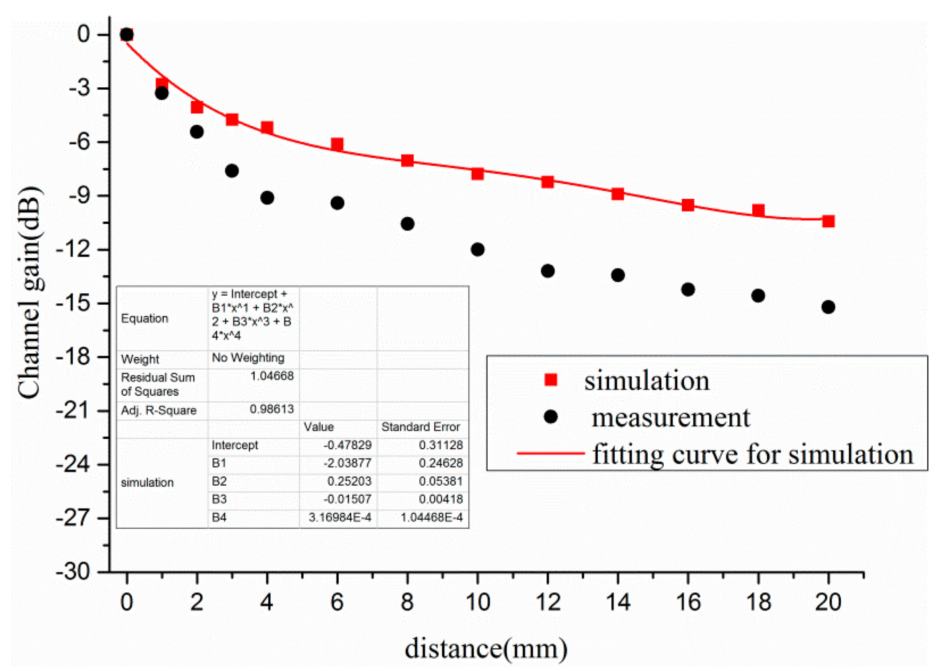

Figure 8. Influence of distance between electrode and human body at Scenario 3.

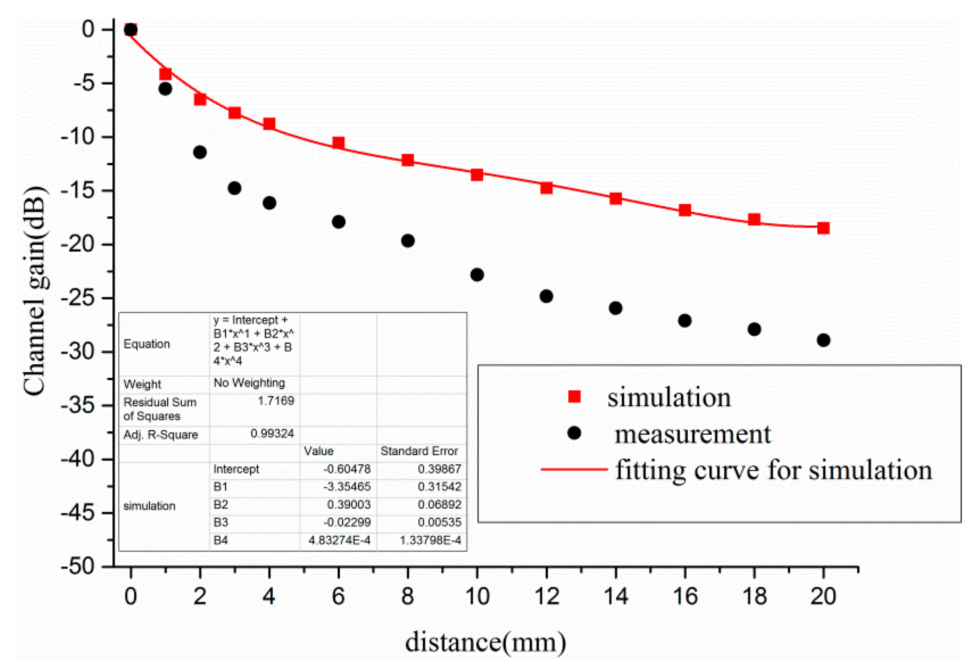

Figure 9. Influence of distance between electrode and human body at Scenario 4 .

\subsection{Discussion}

The channel gain shown in Figure 5 revealed that the channel gain of different scenarios varied with the frequency. Furthermore, due to the influence of the human body, the channel gain of Scenario 2, Scenario 3, and Scenario 4 was much greater than the channel gain of Scenario 1 when the frequency was below $90 \mathrm{MHz}$. Thus, it was indicated that the human body could be used as a transmitting antenna or receiving antenna to achieve the signal transmission, which was helpful to improve the channel gain. However, the difference of channel gain among the four scenarios became small when the frequency was beyond $65 \mathrm{MHz}$, which might be explained by the relationship of power propagation and absorption. As the frequency increased, the power was more easily transmitted through air. Moreover, there was no power absorption by the human body in Scenario 1. Thus, the channel gain of Scenario 1 increased sharply. On the other hand, the power was absorbed by the human body in 
Scenario 2, Scenario 3 and Scenario 4 . Therefore, the difference of the four scenarios became small when the frequency was beyond $65 \mathrm{MHz}$.

On the other hand, as demonstrated in Figure 5, the channel gain of Scenario 2 and Scenario 3 was almost the same in the frequency range $1 \mathrm{MHz}$ to $90 \mathrm{MHz}$, which might be explained by the reciprocity theorem of antenna $[30,31]$. According to the reciprocity theorem, the characteristics remain the same when an antenna is used for transmitting signal or receiving signal under the conduction that the antenna is not nonlinear material. Due to the fact that the human tissues are linear material, the propagation characteristics were similar when the human body was used as a transmitting antenna in Scenario 2 and used as a receiving antenna in Scenario 3.

\section{Conclusions}

This paper presented the propagation characteristics of four scenarios through numerical simulations with an inhomogeneous model and in-situ experiments. Compared with the channel gain of Scenario 1 in which both the TX electrode and RX electrode were placed in the air, the results showed that the channel gain was greater when the TX electrode or RX electrode was attached to the human body in the frequency range $1 \mathrm{MHz}$ to $90 \mathrm{MHz}$. Therefore, it could be inferred that the human body could be regarded as a transmitting antenna or receiving antenna to achieve the signal transmission. Specifically, the input efficiency of Scenario 2 was up to $7.53 \%$ at $44 \mathrm{MHz}$ when the human body was used as a transmitting antenna, whereas the input efficiency of Scenario 1 was just $9.22 \times 10^{-4 \%}$ without the human body. Similarly, the accepted efficiency of Scenario 3 rose from $4.90 \times 10^{-3} \%$ to $1.24 \%$ when the human body was utilized as a receiving antenna. Furthermore, in terms of the electric field distribution, it could be observed that the electric field intensity around the human body was enhanced dramatically when TX electrode or RX electrode was attached on the human body, which also revealed the feasibility of using the human body as a transmitting antenna or receiving antenna. In addition, the factors affecting propagation characteristics were explored when the human body was used as an antenna in this paper. The results indicated that the high-water-content tissues, such as skin, muscle, heart, and so on, played an important role in signal propagation, which was helpful to improve the channel gain. The channel gain of Scenario 3 was $0 \mathrm{~dB}, 1.285 \mathrm{~dB}, 2.521 \mathrm{~dB}$, $-0.871 \mathrm{~dB}$, and $-0.871 \mathrm{~dB}$, respectively when the RX electrode was attached to different position of the human body. In addition, the channel gain of Scenario 4 was $0 \mathrm{~dB},-0.492 \mathrm{~dB},-1.283 \mathrm{~dB},-1.816 \mathrm{~dB}$, $-1.815 \mathrm{~dB},-1.921 \mathrm{~dB}$, and $-1.921 \mathrm{~dB}$ at different electrode positions. Therefore, it seemed to indicate that the channel gain was insensitive with electrode position when the human body was employed as an antenna. However, the channel gain was associated with the distance between electrode and human body. As the distance increased, the channel gain decreased rapidly, and the relationship could be represented by a quartic polynomial. In summary, the human body can be used as an antenna to achieve wireless transmission, which may provide a promising communication solution in BSN with the advantages of low cost and convenience. In the near future, we will investigate the influence of the dynamic human body when it is used as an antenna to achieve wireless transmission.

Acknowledgments: This work was supported by the National Natural Science Foundation of China under Grant No. 61403366 and No. U1505251, Guangdong Science and Technology Planning Project under Grant No. 2015A020214018 and No. 2015B020233004, Shenzhen Technology Development Project Fund under Grant No. CXZZ20150505093829778, Shenzhen Project under Grant No. JSGG20160509142926253, and Shenzhen Public Technology Service Platform Improvement Project of Biomedical Electronics.

Author Contributions: Jingzhen Li performed the numerical simulations and wrote the paper. Zedong Nie provided the initial idea of this research. Yuhang Liu performed the experiments and worked for the data collection and analysis. Lei Wang modified the grammar. Yang Hao provided lots of useful comments in this paper.

Conflicts of Interest: The authors declare no conflict of interest. 


\section{References}

1. Moore, S.M.; McIntosh, R.L.; Iskra, S.; Wood, A.W. Modeling the effect of adverse environmental conditions and clothing on temperature rise in a human body exposed to radio frequency electromagnetic fields. IEEE Trans. Biomed. Eng. 2015, 62, 627-637. [CrossRef] [PubMed]

2. Mobashsher, A.T.; Abbosh, A.M. Artificial human phantoms: Human proxy in testing microwave apparatuses that have electromagnetic interaction with the human body. IEEE Microw. Mag. 2015, 16, 42-62. [CrossRef]

3. Ferikoğlu, A.; Çerezci, O.; Kahriman, M.; Yener, Ş.Ç. Electromagnetic absorption rate in a multilayer human tissue model exposed to base-station radiation using transmission line analysis. IEEE Antennas Wirel. Propag. Lett. 2014, 13, 903-906. [CrossRef]

4. Ibrani, M.; Ahma, L.; Hamiti, E. Assessment of the exposure of children to electromagnetic fields from wireless communication devices in home environments. IET Commun. 2014, 8, 2222-2228. [CrossRef]

5. Kibret, B.; Teshome, A.K.; Lai, D.T.H. Cylindrical antenna theory for the analysis of whole-body averaged specific absorption rate. IEEE Trans. Antennas Propag. 2015, 63, 5224-5229. [CrossRef]

6. Chrissoulidis, D.P.; Laheurte, J.M. Radiation from an encapsulated Hertz dipole implanted in a human torso model. IEEE Trans. Antennas Propag. 2016, 64, 4984-4992. [CrossRef]

7. Vallauri, R.; Bertin, G.; Piovano, B.; Gianola, P. Electromagnetic field zones around an antenna for human exposure assessment: Evaluation of the human exposure to EMFs. IEEE Antennas Propag. Mag. 2015, 57, 53-63. [CrossRef]

8. Gabriel, C.; Gabriel, S.; Corthout, E. The dielectric properties of biological tissues: I. Literature survey. Phys. Med. Biol. 1996, 41, 2231-2249. [CrossRef] [PubMed]

9. Gabriel, S.; Lau, R.; Gabriel, C. The dielectric properties of biological tissues: II. Measurements in the frequency range $10 \mathrm{~Hz}$ to $20 \mathrm{GHz}$. Phys. Med. Biol. 1996, 41, 2251-2269. [CrossRef] [PubMed]

10. Gabriel, S.; Lau, R.; Gabriel, C. The dielectric properties of biological tissues: III. Parametric models for the dielectric spectrum of tissues. Phys. Med. Biol. 1996, 41, 2271-2293. [CrossRef] [PubMed]

11. King, R.W.P. Electric fields induced in cells in the bodies of amateur radio operators by their transmitting antennas. IEEE Trans. Microw. Theory Tech. 2000, 48, 2155-2158. [CrossRef]

12. King, R.W.P. Electric current and electric field induced in the human body when exposed to an incident electric field near the resonant frequency. IEEE Trans. Microw. Theory Tech. 2000, 48, 1537-1543. [CrossRef]

13. Zhu, X.Q.; Guo, Y.X.; Wu, W. Investigation and modeling of capacitive human body communication. IEEE Trans. Biomed. Circuits Syst. 2017, 11, 474-482. [CrossRef] [PubMed]

14. Chen, X.M.; Pun, S.H.; Zhao, J.F.; Mak, P.U.; Liang, B.D.; Vai, M.I. Effects of human limb gestures on galvanic coupling intra-body communication for advanced healthcare system. Biomed. Eng. Online 2016, 15, 60. [CrossRef] [PubMed]

15. Callejon, M.; Reina-Tosina, J.; Naranjo, D.; Roa, L.M. Measurement issues in galvanic intrabody communication: Influence of experimental setup. IEEE Trans. Biomed. Eng. 2015, 62, 2724-2732. [CrossRef] [PubMed]

16. Amparo Callejon, M.; Reina-Tosina, J.; Naranjo-Hernandez, D.; Roa, L.M. Galvanic coupling transmission in intrabody communication: A finite element approach. IEEE Trans. Biomed. Eng. 2014, 61, 775-783. [CrossRef] [PubMed]

17. Kibret, B.; Seyedi, M.; Lai, D.T.; Faulkner, M. Investigation of galvanic-coupled intrabody communication using the human body circuit model. IEEE J. Biomed. Health Inform. 2014, 18, 1196-1206. [CrossRef] [PubMed]

18. Hwang, J.H.; Hyoung, C.H.; Park, K.H.; Kim, Y.T. Energy harvesting from ambient electromagnetic wave using human body as antenna. Electron. Lett. 2013, 49, 149-151. [CrossRef]

19. Simba, A.Y.; Itou, A.; Hamada, L.; Watanabe, S.; Arima, T.; Uno, T. Development of liquid-type human-body equivalent antennas for induced ankle current measurements at VHF band. IEEE Trans. Electromagn. Compat. 2012, 54, 565-573. [CrossRef]

20. Kibret, B.; Teshome, A.K.; Lai, D.T.H. Characterizing the human body as a monopole antenna. IEEE Trans. Antennas Propag. 2015, 63, 4384-4392. [CrossRef]

21. Kibret, B.; Teshome, A.K.; Lai, D.T.H. Analysis of the human body as an antenna for wireless implant communication. IEEE Trans. Antennas Propag. 2016, 64, 1466-1476. [CrossRef] 
22. Frederick, D.A.; Jenkins, B.N. Height and body mass on the mating market: Associations with number of sex partners and extra-pair sex among heterosexual men and women aged 18-65. Evol. Psychol. 2015, 13, 113-115. [CrossRef]

23. Li, J.; Nie, Z.; Liu, Y.; Wang, L.; Hao, Y. Characterization of in-body radio channels for wireless implants. IEEE Sens. J. 2017, 17, 1528-1537. [CrossRef]

24. Lučev, Ž.; Krois, I.; Cifrek, M. A capacitive intrabody communication channel from $100 \mathrm{kHz}$ to $100 \mathrm{MHz}$. IEEE Trans. Instrum. Meas. 2012, 61, 3280-3289. [CrossRef]

25. Xu, R.; Zhu, H.; Yuan, L. Characterization and analysis of intra-body communication channel. In Proceedings of the 2009 IEEE Antennas and Propagation Society International Symposium and USNC/URSI National Radio Science Meeting, North Charleston, SC, USA, 1-5 June 2009; pp. 1-4.

26. Fang, B.; Yi, W.; Chu, C.; Ying, L.; Na, L.; Liu, K.; Tan, L.; Zhang, S. Creation of a virtual anatomy system based on chinese visible human data sets. Surg. Radiol. Anat. 2016, 39, 441-449. [CrossRef] [PubMed]

27. Xu, R.; Zhu, H.; Yuan, J. Electric-field intrabody communication channel modeling with finite-element method. IEEE Trans. Biomed. Eng. 2011, 58, 705-712. [PubMed]

28. Schwan, H.P. Analysis of dielectric data: Experience gained with biological materials. IEEE Trans. Dielectr. Electr. Insul. 1985, 6, 913-922. [CrossRef]

29. Magill, M.K.; Conway, G.A.; Scanlon, W.G. Robust implantable antenna for in-body communications. In Proceedings of the 2015 Loughborough Antennas \& Propagation Conference, Loughborough, UK, 2-3 November 2015; pp. 1-4.

30. Štumpf, M. Pulsed EM field radiation, mutual coupling, and reciprocity of thin planar antennas. IEEE Trans. Antennas Propag. 2014, 62, 3943-3950. [CrossRef]

31. Ling, F.; Jin, J.M. Scattering and radiation analysis of microstrip antennas using discrete complex image method and reciprocity theorem. Microw. Opt. Technol. Lett. 1997, 16, 212-216. [CrossRef]

(C) 2017 by the authors. Licensee MDPI, Basel, Switzerland. This article is an open access article distributed under the terms and conditions of the Creative Commons Attribution (CC BY) license (http:/ / creativecommons.org/licenses/by/4.0/). 